\title{
Long-term and low-dose of busulfan is a safe and effective second-line treatment in elderly patients with essential thrombocythemia resistant or intolerant to hydroxyurea
}

\author{
Rossella Renso ${ }^{1,2}$, Andrea Aroldi ${ }^{1,2}$, Pietro Pioltelli ${ }^{1}$, Carlo Gambacorti-Passerini ${ }^{1,2,3}$ and Elena Maria Elli ${ }^{1}$
}

Essential thrombocythemia (ET) is a Philadelphia negative myeloproliferative neoplasm (MPN) characterized by an increased risk of thrombosis and, in the long-term, transformation to myelofibrosis (MF) or acute leukemia ${ }^{1}$. The purpose of therapy is to achieve a good cytoreduction and to prevent thrombosis and bleeding complications without increasing the transformation risk. In patients at high risk of thrombosis, cytoreduction with hydroxyurea $(\mathrm{HU})$ is currently recommended as first-line therapy ${ }^{2}$. Busulfan (BU) is an alkylating agent that has been used since 1959 for the treatment of $\mathrm{MPN}^{3}$. In some retrospective studies, its use according to the conventional schedule has been associated to high risk of leukemic transformation and second neoplasms, as with other alkylating agents. Consequently, BU is currently used by many hematologists as second-line treatment in Bcr-Abl negative MPN that are resistant to or developed side effects from $\mathrm{HU}$, principally in elderly patients, for whom therapeutic options remain limited ${ }^{4-6}$. The conventional schedule provides a starting dose of $14 \mathrm{mg} /$ week up to obtain a complete haematological response (CHR), defined according to the European LeukemiaNet (ELN) criteria ${ }^{7}$.

In the current retrospective study, we wanted to share our experience with use of long-term and low-dose of BU in elderly patients with ET who are resistant or intolerant to $\mathrm{HU}$.

\footnotetext{
Correspondence: Elena Maria. Elli (elena.elli@libero.it)

${ }^{1}$ Hematology Division and Bone Marrow Unit, San Gerardo Hospital, Monza, Italy

${ }^{2}$ Department of Medicine, University of Milano-Bicocca, Monza, Italy

Full list of author information is available at the end of the article
}

From 1995 to 2015 we collected 348 patients who received ET diagnosis at our department, according to WHO criteria ${ }^{1}$. The principal clinical and hematological features of entire cohort of ET patients were shown in Table 1 . In this population we identified 30 patients who received a second-line treatment with an alternative schedule of BU, defined by low-starting dose $(4-6 \mathrm{mg} /$ week) down to obtain CHR, followed by a dose deescalation of $\mathrm{BU}$ over time up to the minimal dose required for the maintenance of $\mathrm{CHR}$. The dose deescalation of $\mathrm{BU}$ was performed every 4 weeks and the minimal dose to maintain the CHR was generally between $2 \mathrm{mg} /$ week and $2 \mathrm{mg} / \mathrm{month}$.

We analyzed efficacy, toxicity and risk of leukemic evolution in these settings of patients. Intolerance and resistance to $\mathrm{HU}$ were categorized according to ELN criteria for $\mathrm{ET}^{8}$. Non-parametric tests, such as Mann-Whitney, Pearson Chi-square and Fischer's exact tests, were used for statistical analysis of continuous and categorical variables. Leukemia-free-survival (LFS) curves were calculated by Kaplan-Meier method and compared with Log-rank (Mantel-Cox) test.

Twenty-six of 30 patients were evaluable for full demographic, diagnostic and therapeutic information and were considered for statistical analysis. As reported in Table 1, we observed some significant differences between $\mathrm{BU}$ patients and the entire series of ET patients with respect to age and blood counts at diagnosis. The BU patients were older at diagnosis and presented higher platelet (PLT) count and lower levels of hematocrit (Ht), as compared to global ET population. These demographic 
Table 1 Main clinical and hematological features of entire series of patients with essential thrombocythemia (ET) ( $n=$ $348)$ and of patients treated with $(n=26)$ or without $(n=322)$ Busulfan

\begin{tabular}{|c|c|c|c|c|}
\hline Variables analyzed & Busulfan $(n=26)$ & No Busulfan ( $n=322)$ & $\begin{array}{l}\text { Entire cohort ET } \\
(n=348)\end{array}$ & $\begin{array}{l}P \text {-value "Busulfan" vs. } \\
\text { "no Busulfan" }\end{array}$ \\
\hline Gender (male/female) & $7 / 19$ & 131/191 & $138 / 210$ & $P=0.17$ \\
\hline \multicolumn{5}{|l|}{ Driver mutations ( $(\%))$ : } \\
\hline JAK2V617F mutation & $15(57.7 \%)$ & $201(62.4 \%)$ & $216(62.1 \%)$ & $P=0.79$ \\
\hline Calreticulin mutation & $8(30.8 \%)$ & $60(18.6 \%)$ & $68(19.5 \%)$ & $P=0.21$ \\
\hline MPL mutation & $1(3.8 \%)$ & $11(3.4 \%)$ & $12(3.5 \%)$ & $P=0.91$ \\
\hline Triple negative & $2(7.7 \%)$ & $50(15.6 \%)$ & $52(14.9 \%)$ & $P=0.28$ \\
\hline Age (years) at diagnosis ${ }^{a}$ & $71.2(52.7-85.2)$ & $58.7(12.1-92.1)$ & $60.5(12.1-92.1)$ & $P<0.001$ \\
\hline \multicolumn{5}{|l|}{ Blood cell count at diagnosis ${ }^{a}$} \\
\hline Hematocrit (\%) & $40.1(28.6-50.4)$ & $42(29.3-55.5)$ & $42(28.6-55.5)$ & $P=0.028$ \\
\hline Platelets count $\left(\times 10^{9} / \mathrm{L}\right)$ & $788.5(507-2887)$ & $674.5(233-2240)$ & $679.5(233-2887)$ & $P=0.007$ \\
\hline Leukocyte count $\left(\times 10^{9} / \mathrm{L}\right)$ & $8.3(4.84-25.49)$ & $8.4(4.04-28.84)$ & $8.4(4.04-28.84)$ & $P=0.19$ \\
\hline Thrombosis at diagnosis $(n(\%))$ : & 0 & $13(4 \%)$ & $13(3.4 \%)$ & $P=0.61$ \\
\hline Arterial & & $13(4 \%)$ & $13(3.4 \%)$ & \\
\hline Venous & & 0 & 0 & \\
\hline $\begin{array}{l}\text { Thrombosis during follow-up ( } n \\
(\%) \text { : }\end{array}$ & $6(23 \%)$ & $32(9.9 \%)$ & $38(10.9 \%)$ & $P=0.08$ \\
\hline Arterial & $4(15 \%)$ & $26(8.1 \%)$ & $30(8.6 \%)$ & $P=0.26$ \\
\hline Venous & $2(8 \%)$ & $6(1.8 \%)$ & $8(2.3 \%)$ & $P=0.11$ \\
\hline
\end{tabular}

${ }^{\mathrm{a}}$ Median (range)

and laboratory features could reflect some biological differences in disease aggressiveness. Therefore, the clinical outcome was not comparable in term of survival and myelofibrosis evolution.

At the beginning of $\mathrm{BU}$ treatment, median age of patients was 79 years (range 59-88), median $\mathrm{Ht}$ value, white blood cell count (WBC) and PLT count were $39.8 \%$ (range: $30.9-50.6$ ), $6.6 \times 10^{9} / \mathrm{L}$ (range: $3.5-35.1$ ) and $706 \times 10^{9} / \mathrm{L}$ (range: $240-1343$ ), respectively. Median time elapsed from diagnosis to BU start was 3.7 years (range: $0.2-21.2)$. At time of the switch to second-line therapy with BU, no patient was in CHR: 11 patients (42.3\%) were intolerant and 15 (57.7\%) were resistant to HU. Patients received BU for a median time of 48 months (3.7-102.2), with a median cumulative BU dose of $481 \mathrm{mg}$ (80-1032).

Twenty-four of 26 (92.3\%) evaluable patients obtained CHR, after a median time of 7.4 months of drug exposition (range: 3.2-37.4). The median duration of CHR was 43.2 months (range 10-81.9).

Four $(15.4 \%)$ patients presented with hematological adverse events (evaluated according to the National Cancer Institute Common Terminology Criteria for Adverse Events (CTCAE), version 4.0): one patient with grade 3 anemia, one case of grade 3 thrombocytopenia and two patients with grade 2 neutropenia. $75 \%(3 / 4)$ of them discontinued definitively the drug, with a reversible hematological toxicity over 6 months. Only one case of non-hematological cutaneous grade 2 adverse event was documented.

At the time of analysis, 16 (61.5\%) patients stopped BU: 3 for grade $\geq 2$ hematological toxicity, 4 for disease progression, 3 for drug extra-hematological intolerance or resistance, 2 for grade $\geq 2$ infectious complications; the remaining 4 not for drug-related raisons (principally due to personal patient's decision). Fourteen of 16 patients (87.5\%) stopped BU treatment after achievement of CHR.

With a median follow-up of 130 months (range 16-333.3) from diagnosis and 72,7 months (range 5-105.2) from BU start, 6 (23\%) patients have died. Causes of death were disease progression in 3 patients $(n$ $=2$ leukemic evolution, $n=1$ MF transformation), infections $(n=1)$ and unknown $(n=2)$. Thrombotic complications after BU start were observed in 5 (19.2\%) patients ( 3 arterial and 2 venous thrombosis). All patients received anti-platelet therapy associated to BU cytoreduction, according to standard guidelines of treatment for $\mathrm{ET}^{2}$. No major bleedings or second neoplasms were recorded. 


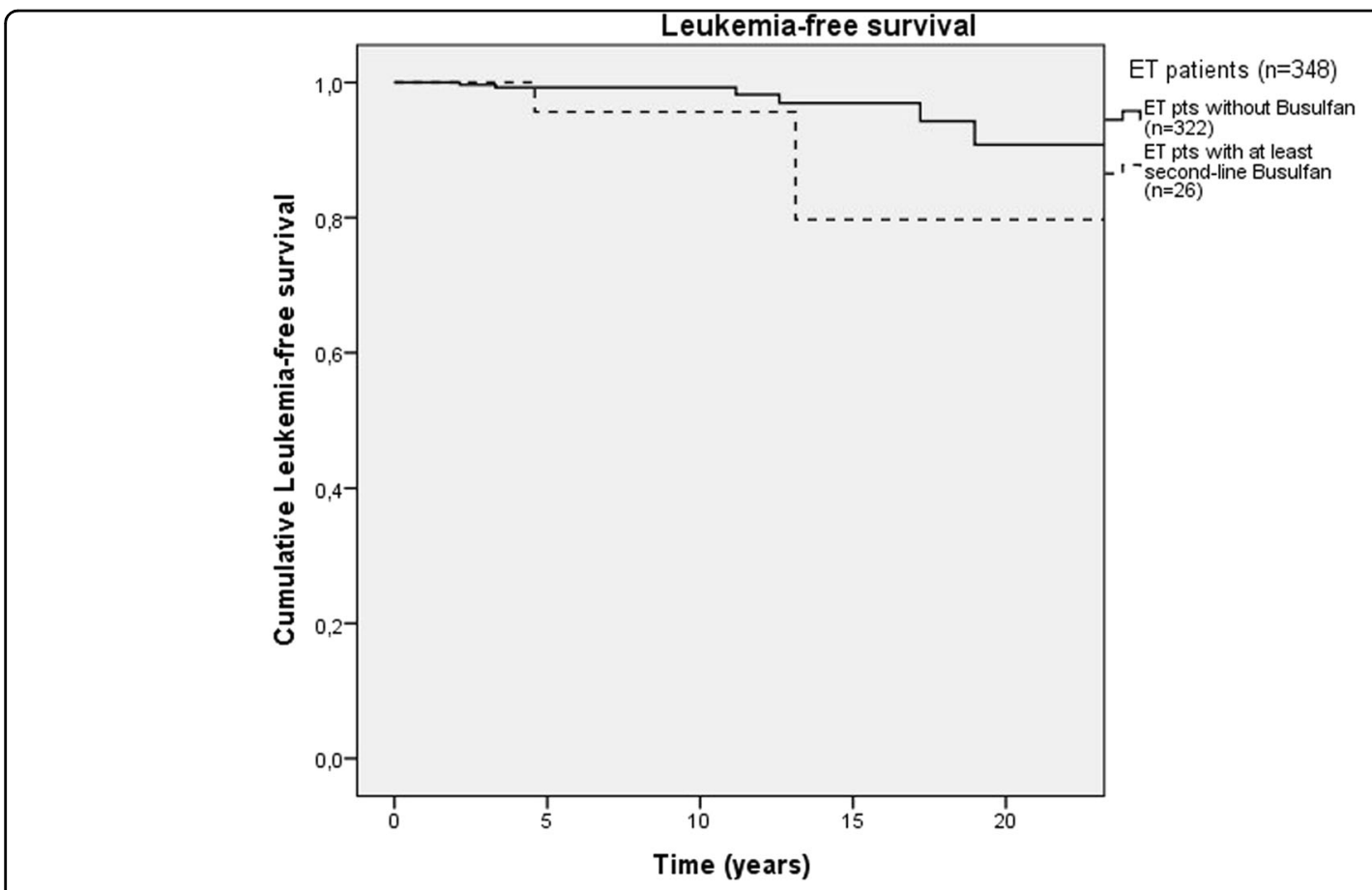

Fig. 1 Leukemia-free survival (LFS) in patients (pts) with Essential Thrombocythemia (ET) treated with Busulfan ( $n=26)$ and without Busulfan ( $n=$ 322)

Leukemic evolution was observed in 2 (7.7\%) patients after a median time of 31.5 months from BU start and 104.8 months from diagnosis. Despite the demographic and biological differences in disease aggressiveness of $\mathrm{BU}$ patients, this incidence of transformation into acute leukemia was similar to the entire series of ET patients (2.6\%, $P=0.14$ ). The LFS at 15 years was comparable in 2 groups ( 97 vs. $79.7 \%$ at 15 years; Fig. $1, P=0.12$ ).

In conclusion, to our knowledge, the present study represents the first experience with an alternative longterm and low dose administration of BU in elderly patients with ET who are resistant or intolerant to HU. This different schedule seems to be safe and effective. It provides a high rate of hematological response $(92.3 \%$ of patients obtained CHR) with acceptable hematological and extrahematological toxicity. The achievement of CHR is rapid (6.5 months) and sustained over time (43.2 months). The risk of leukemic transformation seems to be limited and similar to that is reported in recent literature ${ }^{4,5}$, considering that these patients are very old and previously treated $^{3,4}$. Additional data from larger retrospective multicenter studies or prospective series should be further obtained to confirm the long-term safety and efficacy of this alternative schedule of BU in ET patients.

\section{Author details}

${ }^{1}$ Hematology Division and Bone Marrow Unit, San Gerardo Hospital, Monza, Italy. ${ }^{2}$ Department of Medicine, University of Milano-Bicocca, Monza, Italy. ${ }^{3}$ Hematology Division and Clinical Research Unit, San Gerardo Hospital, Monza, Italy
Conflict of interest

The authors declare that they have no conflict of interest.

\section{Publisher's note}

Springer Nature remains neutral with regard to jurisdictional claims in published maps and institutional affiliations.

Received: 1 January 2018 Revised: 22 April 2018 Accepted: 27 April 2018 Published online: 11 June 2018

\section{References}

1. Arber, D. A. et al. The2016 revision to the World Health Organization classification of myeloid neoplasms and acute leukemia. Blood 127, 2391-2405 (2016).

2. Tefferi, A. \& Barbui, T. Polycythemia vera and essential thrombocythemia: 2017 update on diagnosis, risk-stratification, and management. Am. J. Hematol. 92, 94-108 (2017).

3. Brodsky, I. Busulfan versus hydroxyurea in the treatment of polycythemia vera (PV) and essential thrombocythemia (ET). Am. J. Clin. Oncol. 21, 105-106 (1998).

4. Alvarez-Larrán, A. et al. Busulfan in patients with polycythemia vera or essential thrombocythemia refractory or intolerant to hydroxyurea. Ann. Hematol. 93 2037-2043 (2014).

5. Begna, K. et al. Busulfan for the treatment of myeloproliferative neoplasms: the Mayo Clinic experience. Blood Cancer J. 6, e427 (2016).

6. Douglas, G. et al. Busulfan is effective second-line therapy for older patients with Philadelphia-negative myeloproliferative neoplasms intolerant of or unresponsive to hydroxyurea. Leuk. Lymphoma 58, 89-95 (2017).

7. Barosi, G. et al. Revised response criteria for polycythemia vera and essential thrombocythemia: an ELN and IWG-MRT consensus project. Blood 121, 4778-4781 (2013).

8. Hernández-Boluda, J.-C. et al. Clinical evaluation of the European LeukemiaNet criteria for clinic haematological response and resistance/intolerance to hydroxycarbamide in essential thrombocythemia. Br. J. Haematol. 152, 81-88 (2011) 